\title{
Thermal Error Modeling of a Machine Tool Using Data Mining Scheme*
}

\author{
Kun-Chieh WANG** and Pai-Chang TSENG*** \\ **Department of Technological Product Design, Ling Tung University \\ 1,Lingtung Rd., Nantun, 408 Taichung City, Taiwan \\ E-mail: kcwang@mail.Itu.edu.tw \\ ${ }^{* * \star}$ Depatment of Mechanical Engineering, National Chung Hsing University \\ 250, Kuo Kuang Rd., 402 Taichung City, Taiwan
}

\begin{abstract}
In this paper the knowledge discovery technique is used to build an effective and transparent mathematic thermal error model for machine tools. Our proposed thermal error modeling methodology (called KRL) integrates the schemes of K-means theory (KM), rough-set theory (RS), and linear regression model (LR). First, to explore the machine tool's thermal behavior, an integrated system is designed to simultaneously measure the temperature ascents at selected characteristic points and the thermal deformations at spindle nose under suitable real machining conditions. Second, the obtained data are classified by the KM method, further reduced by the RS scheme, and a linear thermal error model is established by the LR technique. To evaluate the performance of our proposed model, an adaptive neural fuzzy inference system (ANFIS) thermal error model is introduced for comparison. Finally, a verification experiment is carried out and results reveal that the proposed KRL model is effective in predicting thermal behavior in machine tools. Our proposed KRL model is transparent, easily understood by users, and can be easily programmed or modified for different machining conditions.
\end{abstract}

Key words: Thermal Error Compensation, K-means Method, Rough Set Theory, Artificial Intelligence, Machine Tools

\section{Introduction}

Thermal error is responsible for as much as $60-70 \%$ of the total error in a machine tool during operation. Although part of the thermal error can be reduced through methods such as structural improvement or heat-source isolation, their effectiveness is still limited. An effective method to address this issue is the adoption of thermal error compensation. The methodology of this scheme aims to perform heat error forecasting for machine tools by measuring the temperature ascent and thermal deformation in given machining conditions, and then applying certain theories and rules to analyze the experimental results, and thereby predict the machine's thermal deformation in processing. Particularly, it is noted that the performance of a thermal error compensation system significantly depends on the thermal error model used. The thermal error model becomes one of the most critical factors in compensating for errors generated in machine tools on account of the temperature ascent of the various machine tool elements. 
Extensive research has been carried out in the past in the area of thermal error modeling (1-19). There are two main research areas related to thermal error modeling. The first area is numerical analysis techniques such as the finite element method ${ }^{\mathbf{1})(2)}$ and finite difference method $^{(3)}$. These techniques are restricted to a qualitative analysis because the boundary conditions and heat transfer characteristics cannot be clearly defined. The second area is empirical modeling based on the measurement of thermal deformation and temperature at discrete representative points on the machine tools. Examples include engineering judgment ${ }^{(4)}$, regression analysis ${ }^{(6)}$, neural network ${ }^{(7-9)}$, grey system theory (13), fuzzy logic (16), and adaptive network fuzzy inference system ${ }^{(20)}$, and a combination of feed-forward neural network and hybrid filter schemes ${ }^{(21)}$. Further, some researchers ${ }^{(\mathbf{2 2 , 2 3 )}}$ have remarked upon how the different operating conditions influence the building of thermal error compensation systems.

In summary, empirical models have been demonstrated to show satisfactory predictive accuracy in many applications even though each method has its own limitations. However, some prime difficulties arise. Most of the proposed thermal-error modeling techniques are highly sophisticated and not easy to understand or apply. In addition, it is very difficult and uneconomical to deal with many different machining conditions (including cutting material) in modeling, even more so to build a real-time, accurate thermal error model corresponding to these machining conditions. Furthermore, there is another important problem requiring solution: A great amount of collected measured data is imperfect, ambiguous, ineffective or useless. Using these data in building a thermal error model may cause serious deviations to occur.

This work aims to establish an effective thermal-error modeling technique which satisfies the following requirements: (1) Real cutting conditions should be simple and as few as possible. (2) Measured imperfect and/or ambiguous data should be handled before modeling. (3)The modeling technique should be transparent for easy understanding as well as being easily modifiable to adapt to different usage environments. (4) Modeling results should be accurate and meaningful.

To address this issue, a data mining based integrated scheme, including K-means (KM) theory, rough set (RS) theory, and linear regression (LR) model, was adopted. This integration scheme is called KRL. The development of our proposed KRL scheme is focused on the techniques of data reduction. For demonstration, a C-type vertical CNC machining center is chosen as our application target. Through the manipulation of KRL, a transparent, easy-to-understand, easy-to-program, easily adaptable, and accurate thermal error model is established. A brief explanation of the theories of KM and RS is presented in the next section before showing the details of the model and its features.

\section{Theoretical Bases}

In order to build an effective thermal error model, three different knowledge discovery techniques are included in our study: the K-means theory, the rough set theory, and the linear regression theory. The KM theory is primarily used to classify measured temperature ascents and thermal deformations, to acquire valuable information from these data. The RS theory is used to further handle previously obtained information to obtain the useful knowledge elements in order to build a transparent, accurate, and simple thermal error model. The modeling result expressed in the form of linear regression is essential since it is transparent (easy to understand) to users. Through the LR model, users can quickly perceive the influence weighting of each independent parameter and easily apply this knowledge to further refine the established models in order to adapt to sophisticated cutting conditions during usage. Following are brief introductions of KM and RS theories. 


\subsection{K-means theory}

The KM clustering was brought forward by J. B. McQueen ${ }^{(24)}$ in 1967, which is an early clustering algorithm and a popular method for solving grouping problems. It is easy to practice because its concepts are easy to understand. It aims at looking for points $c_{j}(j=1 \sim k)$, called center or centroid, from $d$-dimension space $(R d)$, and minimizing the square of the distances of $n$ points $p_{i} \quad(i=1 \sim n)$ to its closest centroid (square error), which is also called the $\mathrm{K}$-means error.

The KM clustering is one kind of cutting grouping. Since it must calculate the distances of every data point and its closest controid in each process bout, the calculation time is in positive proportion to the data amount, number of dimensions and number of groups. A good attribute selection institute plays a vitally important role in the quality and efficiency of data mining processing and results.

It is required to designate the number of group $k$ first, and $k \geqq 2$. There is a centroid representing the group itself, which is also the center of the group. The steps of KM clustering are: First, select $k$ items from the data set at random as centroids, then assign each item to its closest centroid according to the Euclid distance between them to form relevant clusters. Second, solve for the new centroid of each cluster then allocate each item to its closest, just generated, controid, repeating this step until no item moves from one cluster to another, i.e. the clustering results approach stability (the centroids also).

For example, we divide the 21 items in into 3 groups $(k=3)$, following the above steps, and choose 3 items at random as centroids, then assign all items to its closest centroid. The formula is

$$
\sum_{i=1}^{n}\left(p_{i}-c_{f(i)}\right)^{2}, f(i)=1 \sim k
$$

where: $c_{f(i)}$ is the centroid of the cluster $p_{i}$ belongs to.

The basic algorithm is:

Step 1 Determining the number of cluster you want $k$ (This is a fixed and artificial value).

Step 2 Generating $k$ centroids at random or by experience $\left(c_{j}, j=1 \sim k\right)$.

Step 3 Allocating $n$ points ( $p_{i}, i=1 \sim n$ ) to the relative closest centroid.

Step 4 Averaging the coordinates of all points of each cluster to make its new centroid.

Step 5 Adding up the square of the distances of each points and its new centroid, i.e. new $k$-means error.

Step 6 Repeating step 3 until satisfying stopping conditions.

The complexity of this method exists in Step 3 because the distances of each point and $k$ centroid should be calculated before the closest centroid is determined.

\subsection{Rough set theory}

The RS theory, first described by Zdzishaw I. Pawlak ${ }^{(25)}$, is a formal approximation of a crisp set (i.e., conventional set) in terms of a pair of sets which give the lower and upper approximation of the original set. In the standard version of rough set theory, the lower and upper approximation sets are crisp, but in other variations, the approximation sets may be fuzzy sets.

\subsubsection{Definitions}

(1) Information system framework

Let $I=(U, A)$ be an information system (attribute-value system), where $U$ is a non-empty set of finite objects (the universe) and $A$ is a non-empty, finite set of attributes such that $a: U \rightarrow V_{a}$ for every $a \in A . V_{a}$ is the set of values that attribute $a$ may take. With any $P \subseteq A$, there is an associated equivalence relation $\operatorname{IND}(P)$ :

$$
\operatorname{IND}(P)=\left\{(x, y) \in U^{2} \mid \forall a \in P, a(x)=a(y)\right\}
$$


The partition of $U$ generated by $\operatorname{IND}(P)$ is denoted $U / I N D(P)$ and can be calculated as follows:

$$
U / \operatorname{IND}(P)=\otimes\{U / \operatorname{IND}(\{a\}) \mid a \in P\}
$$

If $(x, y) \in I N D(P)$, then $x$ and $y$ are indiscernible by attributes from $P$. These indistinguishable sets of objects therefore define an equivalence or indiscernibility relation, referred to as the $P$-indiscernibility relation. The equivalence classes of the $P$-indiscernibility relation are denoted $[x]_{P}$.

\section{(2) Definition of rough set}

Let $X \subseteq U$ be a target set that we wish to represent using attribute subset $P$; and we wish to express this class using the equivalence classes induced by attribute subset $P$. In general, $X$ cannot be expressed exactly, because the set may include and exclude objects which are indistinguishable on the basis of attributes $P$. However, the target set $X$ can be approximated using only the information contained within $P$ by constructing the $P$-lower and $P$-upper approximations of $X$.

$$
\underline{P} X=\left\{x \mid[x]_{P} \subseteq X\right\}, \quad \bar{P} X=\left\{x \mid[x]_{P} \cap X \neq 0\right\}
$$

(3) Lower approximation and positive region

The $P$-lower approximation, or positive region, is the union of all equivalence classes in $[x]_{P}$ which are contained by the target set of $X$. The lower approximation is the complete set of objects in $U / \operatorname{IND}(P)$ that can be positively (i.e., unambiguously) classified as belonging to target set $X$.

(4) Upper approximation and negative region

The $P$-upper approximation is the union of all equivalence classes in $[x]_{P}$ which have non-empty intersection with the target set. The upper approximation is the complete set of objects in $U / I N D(P)$ that cannot be positively (i.e., unambiguously) classified as belonging to the complement of the target set $\bar{X}$. The set $U-\bar{P} X$ therefore represents the negative region, containing the set of objects that can be definitely ruled out as members of the target set.

\section{(5) Boundary region}

The boundary region, given by $\bar{P} X-\underline{P} X$, consists of those objects that can neither be ruled in nor ruled out as members of the target set $X$.

(6) The rough set

The tuple $(\underline{P} X, \bar{P} X)$ composed of the lower and upper approximation is called a rough set; thus, a rough set is composed of two crisp sets, one representing a lower boundary of the target set $X$, and the other representing an upper boundary of the target set $X$. The accuracy of the rough-set representation of the set $X$ can be given by the following:

(7) Definability

$$
\alpha_{P}(X)=|\underline{P} X| /|\bar{P} X|
$$

In general, the upper and lower approximations are not equal; in such cases, we say that target set $X$ is indefinable or roughly definable on attribute set $P$. When the upper and lower approximations are equal, $\underline{P} X=\bar{P} X$, then the target set is definable on attribute $P$.

\section{(8) Reduct and core}

Often we wonder whether there is a subset of attributes which can, by itself, fully characterize the knowledge in the database; such an attribute set is called a reduct. Formally, a reduct is a subset of attributes $R E D \subseteq P$ such that: (a) $[x]_{R E D}=[x]_{P}$, that is, the equivalence classes induced by the reduced attributes set $R E D$ are the same as the 
equivalence class structure induced by the full attribute set $P$, (b) the attribute set $R E D$ is minimal, in the sense that $[x]_{(R E D-\{a\})} \neq[x]_{P}$ for any attribute $a \in R E D$.

The set of attributes which is common to all reducts is called the core: the core is the set of attributes which is possessed by every legitimate reduct, and therefore consists of attributes which cannot be removed from the information system without causing collapse of the equivalence-class structure. The core may be thought of as the set of necessary attributes.

\subsubsection{Rule extraction}

(1) Logical representation of decision tables

Consider an information system:

$$
I=(U, A)=\left(U, P \cup Q, V_{a}\right), \quad U=\left\{O_{1}, O_{2}, \cdots\right\}
$$

in which $O_{i}$ means objects under consideration, $P_{j}$ means attributes belonging to $O_{i}$, and $Q$ means decision attribute. This knowledge system initially may be easily expressed in a tabular form. The table can be transformed into logical form by simply expressing the set of objects as the following (example) disjunction, which corresponds to the disjunctive normal form (DNF) of propositional logics.

$$
\left[\left(P_{1}=1\right) \wedge\left(P_{2}=2\right) \wedge\left(P_{3}=1\right)\right] \vee\left[(P=1) \wedge\left(P_{2}=1\right) \wedge\left(P_{3}=0\right)\right] \vee \cdots
$$

The above form can be written much more compactly by the logical formula:

$$
\left(P_{1}^{1} P_{2}^{2} P_{3}^{1}\right) \vee\left(P_{1}^{1} P_{2}^{1} P_{3}^{0}\right) \vee \cdots
$$

(2) Extracting rules

Our final purpose is to find the minimal set of consistent rules that characterize the system. For a set of condition attributes $P=\left\{P_{1}, P_{2}, \cdots, P_{N}\right\}$ and a decision attribute $Q, Q \notin P$ these rules should have the form

$$
P_{i}^{a} P_{j}^{b} \cdots P_{k}^{c} \rightarrow Q^{d}
$$

where $\{a, b, c\}$ are legitimate values from the domains of their respective attributes. This is a form typical of association rules, and the number of items in $U$ that match the condition/antecedent is called the support for the rule. The method for extracting such rules given in is to form a decision matrix corresponding to each individual value $d$ of decision attribute $Q$. Informally, the decision matrix for value $d$ of decision attribute $Q$ lists all attribute-value pairs that differ between objects having $Q=d$ and $Q \neq d$.

(3)The decision matrix

First, we look at the case $Q=1$, and we separate into objects that have $Q=1$ and those that have $Q \neq 1$. The decision matrix for $Q=1$ lists all the differences between the objects having $Q=1$ and those having $Q \neq 1$.

\section{(4) Writing the rules}

Next, from each decision matrix we form a set of Boolean expressions, one expression for each row of the matrix. The items within each cell are aggregated disjunctively, and the individual cells are then aggregated conjunctively. In general, the procedure will be repeated for each possible value of the decision variable.

\section{Experiment Design}

\subsection{Experiment apparatus}

The experiment apparatus was composed of a CNC vertical machining center (Model Vcenter 205, Victor Taichung Machinery) and sensing units. The experimental sensing apparatus include thermal sensors and a displacement sensor. Semi-conductor type sensors (AD590J) were used as thermal sensors to detect temperature variation in the machine structure due to their advantages of fine calibration, high precision, and easy installation. A 
Renishaw TS27R tool length detection system was used as the displacement sensor for detecting spindle drift error in the Z-axis direction. In measurement, the standard bar quickly moves to the right-up position of the TS27R and then triggers the TS27R probe. After the initial trigger (with feeding rate $100 \mathrm{~mm} / \mathrm{min}$ ), the original touch position (original length of standard bar) can be detected. For the second and sequential triggers, the feeding rate is set at $10 \mathrm{~mm} / \mathrm{min}$ and the positional changes (due to deformation of standard bar or spindle nose) can be detected. The DSP digital signal processor of model TMS320C6713DSK was used for data processing because of its simple structure, high processing speed, easy programming and easy learning curve. The integrated measurement system of temperature and deformation detection as well as signal processing units is demonstrated in Fig.1.

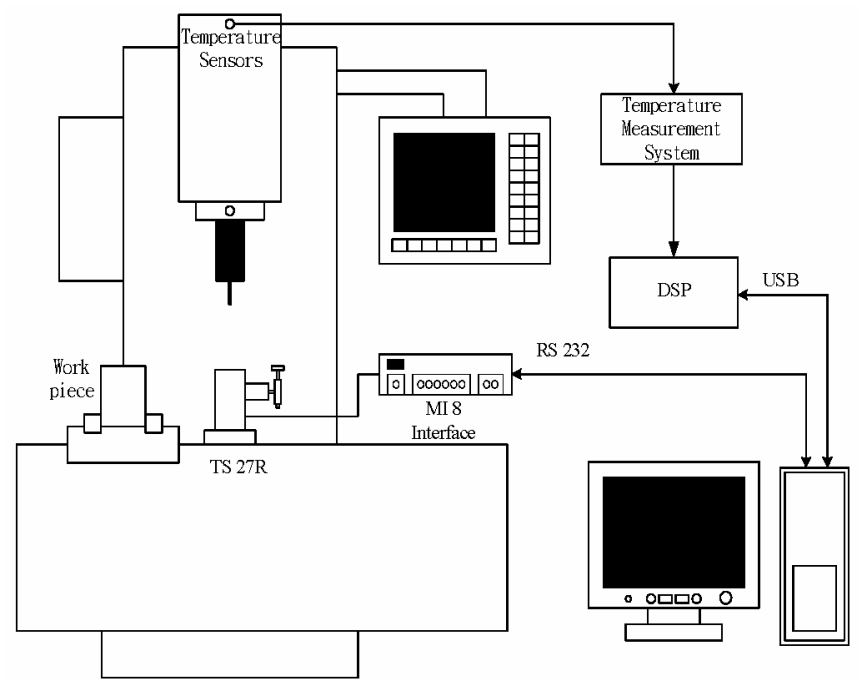

Fig. 1 Schematic diagram of integrated measurement system

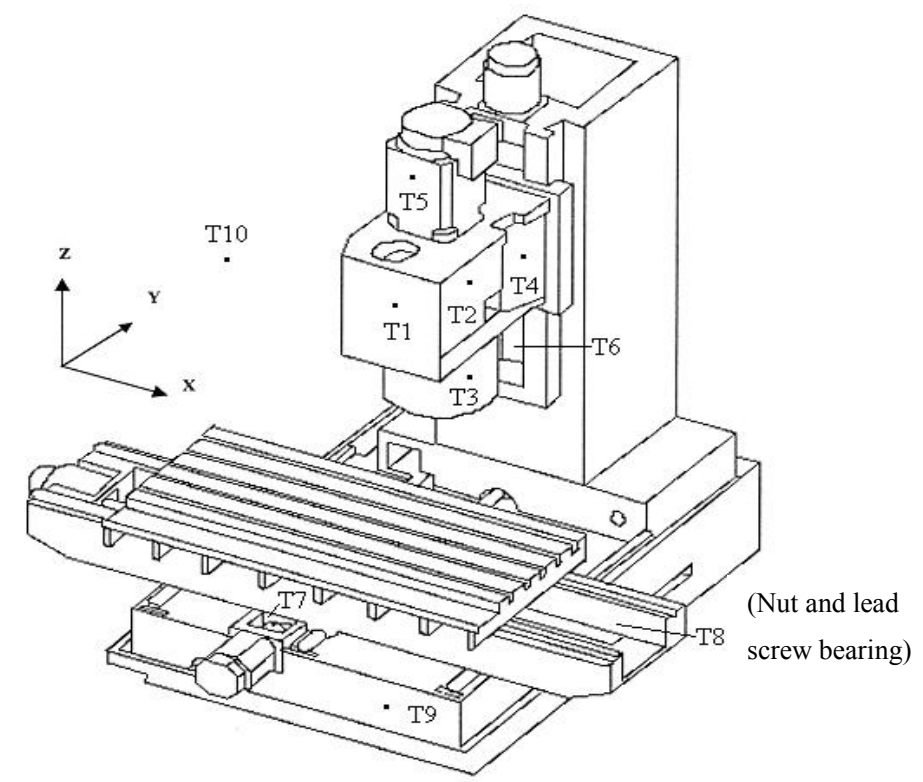

Fig. 2 Characteristic points for temperature ascent measurement

\subsection{Characteristic points}

In total ten temperature sensors were stamped at critical points on the machine, which are known as important heat generation locations to detect temperature ascents, as illustrated in Fig.2. For the $X$-axis motion, sensor $T 8$ measures temperatures on the nut and 
lead screw bearing. For the $Y$-axis motion, $T 7$ measures temperatures on the nut and lead screw bearing. For the $Z$-axis motion, there is no sensor placed on the machine structure since major machine parts here all move relatively slowly compared to those parts of the $X$-axis and $Y$-axis. However, friction effects cannot be ignored since the spindle unit (including the drive motor) may generate significant heat because of relative motion. Thus we choose T6, located at the trail of the machine column, to monitor the effect of heat formation for the $Z$-axis motion. For the machine base, $T 9$ measures temperatures on the machine bed. For the most crucial component for heat generation, the spindle unit, we install five sensors to monitor its temperature at different locations. Among them, sensors T1, T2, and T4 read temperatures on the spindle case; sensors T3 and T5 measure temperatures on the front and rear housings of the spindle motor respectively. Finally, sensor $T 10$ is placed outside the machine to monitor the environmental temperature variations.

It should be noted that too many characteristic points will cause excessive measurement time as well as a great deal of time for modeling computation. On the other hand, too few characteristic points will cause the loss of valuable information about thermal errors. Here, based on engineer's experiences, we carefully choose ten characteristic points in conjunction with the adoption of the RS theory, to handle the measured inaccurate, residual, or grey data at these points. A proper thermal error model being established in this way is much more reliable and efficient.

\subsection{Real-cutting experiment}

To investigate the machinery's thermal behavior, experiments are performed under real-cutting conditions which are fundamental machining processes for users in work-piece machining. These designed real-cutting conditions are listed in Table 1.

Table 1 Real-cutting test conditions.

\begin{tabular}{|c|c|c|c|c|}
\hline Speed & $\begin{array}{c}\text { Cutting } \\
\text { Depth }\end{array}$ & Feed rate & CuttingWay & Work Piece \\
\hline $700(\mathrm{rpm})$ & $3(\mathrm{~mm})$ & $1200(\mathrm{~mm} / \mathrm{min})$ & Zig-Zag & $\begin{array}{c}\text { Medium Carbon } \\
\text { Steel 45C }\end{array}$ \\
\hline
\end{tabular}

\section{Modeling and Discussion}

\subsection{Thermal behavior of machine tool}

The temperature ascents at selected critical points on the machine and the thermal drifts of the spindle nose in the $Z$-axis direction were measured simultaneously using the integrated experiment apparatus and test conditions described previously. The measured results of temperature ascent and thermal deformation are shown in Fig. 3 and Fig. 4 respectively. Fig. 3 shows that the maximum temperature ascent occurs at the spindle motor (T8), reaching nearly $18{ }^{\circ} \mathrm{C}$ after 60 minutes of operation. Fig. 4 shows that maximum thermal drift occurs at the spindle nose in the $Z$-axis direction, which is $83 \mu \mathrm{m}$ after 90 minutes of operation, and then approximately maintains this value when the machine is in a thermally balanced state.

\subsection{Knowledge discovery}

\subsubsection{Data Classification Using KM Method}

There are a total of ten selected critical points for temperature ascent measurements (marked as T1 T10) and 90 data were sampled (recorded every minute) at each critical point. At each critical point, we choose 14 observation time scales (every 5 minutes) as 
sampling bases, marked as $X 1 \sim X 14$. First, ten least-square approximate lines of temperature ascent versus time are obtained using these sampling data. Similarly, one least-square approximate line of thermal deformation versus time is obtained using data shown in Fig. 4. Secondly, the temperature variation $\left(\Delta T_{i}=T_{i}-T_{\text {ref }}\right)$ is obtained by subtraction of the reference temperature $T_{\text {ref }}$ (temperature ascent on the correlation straight line) from $T_{i}$ (measured temperature ascent). Similarly, the deformation change $\left(\Delta \delta_{z i}\right)$, $\Delta \delta_{z i}=\sqrt{\left(\delta_{z i}-\overline{\delta_{z i}}\right)^{2}}$, obtained by a square root of the subtraction of reference deformation $\overline{\delta_{z i}}$ (average deformation on the correlation straight line) from $\delta_{z i}$ (measured thermal deformation), is used as the decision attribute to represent the linearization extent of each temperature ascent. The calculated linearization error results are shown in Table 2.

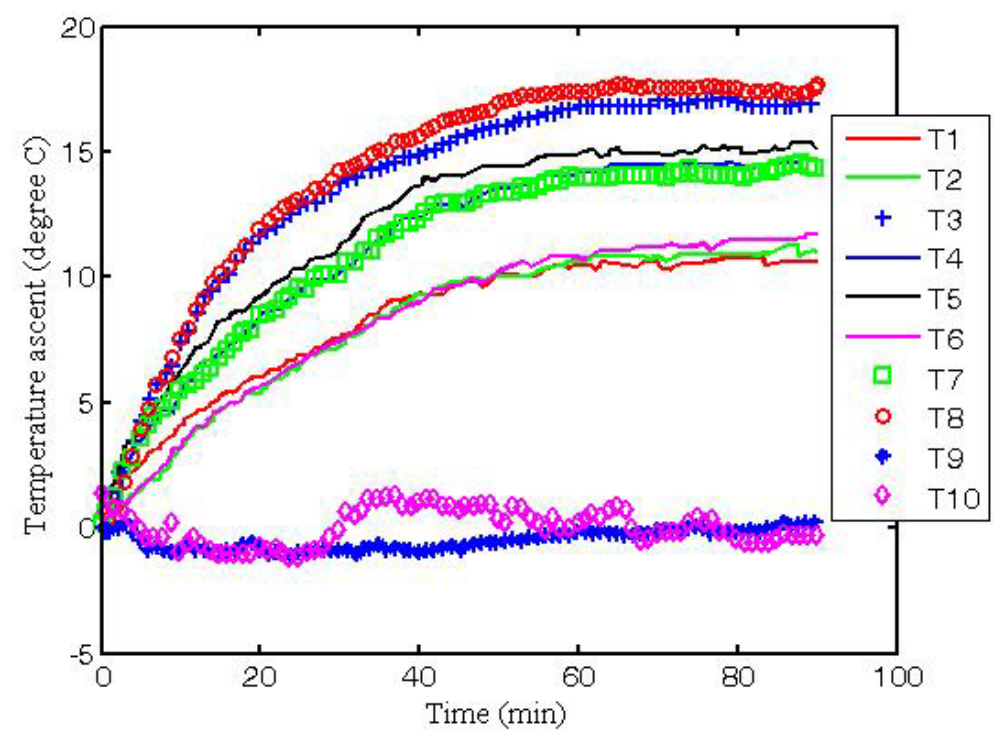

Fig. 3 Variation of temperature ascents at different critical points under real-cutting conditions

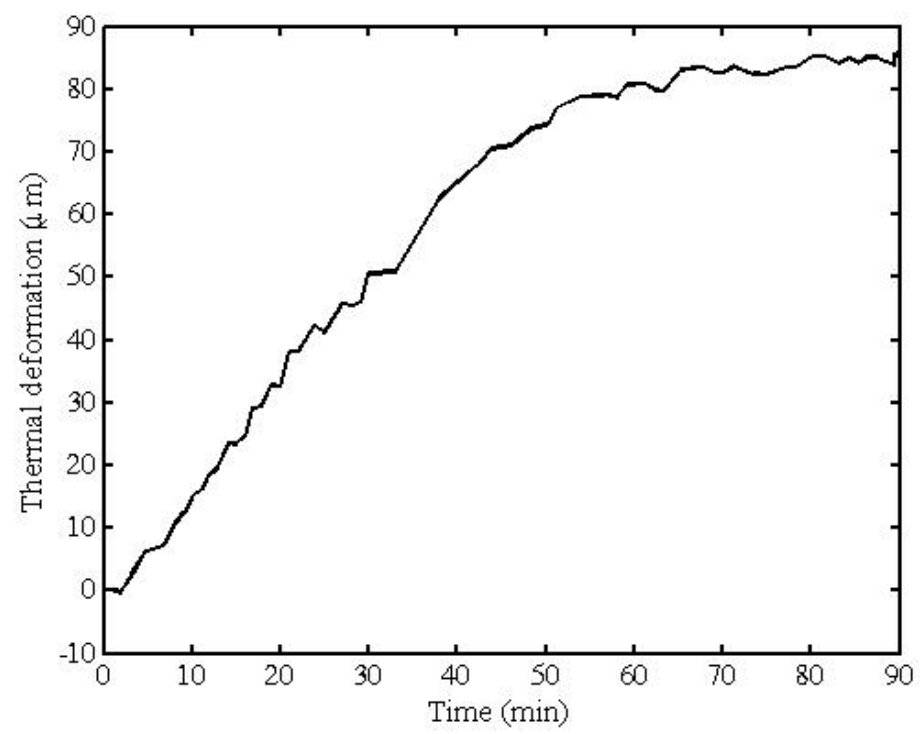

Fig. 4 Thermal error variation of spindle nose in the Z-axis direction under real-cutting conditions. 
To further provide the linearization extent of our measured data, the temperature variation at each characteristic point $\Delta T_{i}$ is classified into 3 4 levels shown in Table 3, according to its magnitude (the level of $\Delta T_{i}$ is marked as $P i$ and called condition attributes). Using the KM data mining scheme, the value of $\Delta \delta_{z i}$ (marked as $Q$ and called as the decision attribute) may be appropriately classified into two groups, represented as "Yes (centered at 4.088)," representing small linearization error, and "No (centered at 12.28)," representing large linearization error, according to its deviation magnitude. The above results can be found in Table 4 .

Apparently, two kinds of sampling time scales are obtained:

(1)Good linearization group of time scales: $X 3, X 4, X 5, X 6, X 10, X 11, X 12$.

(2)Bad linearization group of time scales: $X 1, X 2, X 7, X 8, X 9, X 13, X 14$.

If we want to build a linear thermal error model, the data sampled at the time scales of the first group should be considered as the first priority.

Table 2 Linearization error results at different time scales

\begin{tabular}{lllllllllllll} 
Time(min) & $\Delta T 1$ & $\Delta T 2$ & $\Delta T 3$ & $\Delta T 4$ & $\Delta T 5$ & $\Delta T 6$ & $\Delta T 7$ & $\Delta T 8$ & $\Delta T 9$ & $\Delta T 10$ & $\Delta \delta_{z i}$ \\
\cline { 2 - 11 }$X 1$ & $(5)$ & 0.25 & 0 & 0 & 0.5 & 0.5 & 0.3 & 0.4 & -0.15 & 0 & 1.15 & 12.816 \\
$X 2$ & $(10)$ & 3.125 & 2.125 & 2 & 4.375 & 5 & 4.5 & 5.6 & 5.7 & -0.7 & -0.55 & 12.303 \\
$X 3$ & $(15)$ & 5 & 4.5 & 4.375 & 6.875 & 8 & 6.4 & 9.45 & 9.7 & -1 & -1.12 & 3.519 \\
$X 4$ & $(20)$ & 6.25 & 4.875 & 5.625 & 8.625 & 9.875 & 8.6 & 11.8 & 12.2 & -1 & -1.1 & 3.804 \\
$X 5$ & $(25)$ & 7.25 & 7.125 & 7 & 10 & 11 & 10.1 & 13.25 & 13.6 & -0.9 & -1.12 & 4.212 \\
$X 6$ & $(30)$ & 8.625 & 8.5 & 8.25 & 11.5 & 12.8 & 11.5 & 14.43 & 15 & -0.65 & 1 & 6.68 \\
$X 7$ & $(35)$ & 9.5 & 8.5 & 9.375 & 12.875 & 14 & 12.9 & 15.1 & 15.95 & -0.8 & 0.9 & 11.943 \\
$X 8$ & $(40)$ & 9.875 & 10 & 9.875 & 13.5 & 14.5 & 13.1 & 15.95 & 16.6 & -0.6 & 0.75 & 11.081 \\
$X 9$ & $(45)$ & 10.375 & 10.625 & 10.625 & 14.125 & 14.9 & 13.8 & 16.6 & 17.3 & -0.33 & -0.2 & 9.108 \\
$X 10$ & $(50)$ & 10.5 & 10.9 & 10.9 & 14.2 & 14.875 & 14.05 & 16.81 & 17.5 & -0.13 & 0.3 & 2.783 \\
$X 11(55)$ & 10.5 & 11 & 11 & 14.48 & 15 & 14.1 & 17.05 & 17.45 & -0.1 & -0.4 & 1.284 \\
$X 12$ & $(60)$ & 10.625 & 10.875 & 11 & 14.5 & 15 & 14.05 & 17.05 & 17.85 & -0.2 & 0.2 & 6.336 \\
$X 13$ & $(65)$ & 10.6 & 11.1 & 11.375 & 14.4 & 15.125 & 14.2 & 16.85 & 17.35 & 0.01 & -0.7 & 11.638 \\
$X 14$ & $(70)$ & 10.625 & 11.1 & 11.5 & 14.75 & 15.5 & 14.4 & 16.95 & 17.4 & 0.15 & -0.45 & 17.054
\end{tabular}

Table 3 Range of condition attributes

\begin{tabular}{lllll} 
& level1 & level2 & level3 & level4 \\
\cline { 2 - 5 }$\Delta \boldsymbol{T 1}$ & $(-\infty, 5]$ & $(5,8]$ & $(8, \infty)$ & \\
$\Delta T 2$ & $(-\infty, 4]$ & $(4,8]$ & $(8, \infty)$ & \\
$\Delta T 3$ & $(-\infty, 4]$ & $(4,8]$ & $(10, \infty)$ & \\
$\Delta T 4$ & $(-\infty, 4]$ & $(4,8]$ & $(8,12]$ & {$[12, \infty)$} \\
$\Delta T 5$ & $(-\infty, 4]$ & $(4,8]$ & $(8,12]$ & {$[12, \infty)$} \\
$\Delta T 6$ & $(-\infty, 4]$ & $(4,8]$ & $(8,12]$ & {$[12, \infty)$} \\
$\Delta T 7$ & $(-\infty, 4]$ & $(4,8]$ & $(8,12]$ & {$[12, \infty)$} \\
$\Delta T 8$ & $(-\infty, 4]$ & $(4,8]$ & $(8,12]$ & {$[12, \infty)$} \\
$\Delta T 9$ & $(-\infty,-0.5]$ & $(-0.5,-0.1]$ & $(-0.1, \infty)$ & \\
$\Delta T 10$ & $(-\infty,-0.5]$ & $(-0.5,-0.1]$ & $(-0.1,0.7)$ & {$[0.7, \infty)$}
\end{tabular}


Table 4 Level values of condition and decision attributes at different time scales

\begin{tabular}{lccccccccccc} 
& $\boldsymbol{P 1}$ & $\boldsymbol{P 2}$ & $\boldsymbol{P 3}$ & $\boldsymbol{P 4}$ & $\boldsymbol{P 5}$ & $\boldsymbol{P 6}$ & $\boldsymbol{P 7}$ & $\boldsymbol{P 8}$ & $\boldsymbol{P 9}$ & $\boldsymbol{P 1 0}$ & $\boldsymbol{Q}$ \\
\cline { 2 - 10 } $\boldsymbol{X 1}$ & 1 & 1 & 1 & 1 & 1 & 1 & 1 & 1 & 3 & 4 & NO \\
$X 2$ & 1 & 1 & 1 & 2 & 2 & 2 & 2 & 2 & 1 & 1 & NO \\
$X 3$ & 2 & 2 & 2 & 2 & 2 & 2 & 3 & 3 & 1 & 1 & YES \\
$X 4$ & 2 & 2 & 2 & 3 & 3 & 3 & 3 & 4 & 1 & 1 & YES \\
$X 5$ & 2 & 2 & 2 & 3 & 3 & 3 & 4 & 4 & 1 & 1 & YES \\
$X 6$ & 3 & 3 & 3 & 3 & 4 & 3 & 4 & 4 & 1 & 4 & YES \\
$X 7$ & 3 & 3 & 3 & 4 & 4 & 4 & 4 & 4 & 1 & 4 & NO \\
$X 8$ & 3 & 3 & 3 & 4 & 4 & 4 & 4 & 4 & 1 & 4 & NO \\
$X 9$ & 3 & 3 & 3 & 4 & 4 & 4 & 4 & 4 & 2 & 2 & NO \\
$X 10$ & 3 & 3 & 3 & 4 & 4 & 4 & 4 & 4 & 2 & 3 & YES \\
$X 11$ & 3 & 3 & 3 & 4 & 4 & 4 & 4 & 4 & 2 & 2 & YES \\
$X 12$ & 3 & 3 & 3 & 4 & 4 & 4 & 4 & 4 & 2 & 3 & YES \\
$X 13$ & 3 & 3 & 3 & 4 & 4 & 4 & 4 & 4 & 3 & 1 & NO \\
$X 14$ & 3 & 3 & 3 & 4 & 4 & 4 & 4 & 4 & 3 & 2 & NO
\end{tabular}

\subsubsection{Determination of Linear Attributes Combination Using DM}

According to the previously mentioned RS theory, we now adopt a simple attribute criterion to form the discernibility matrix (DM), as following:

$$
\left\{\begin{array}{cc}
P \in A, P\left(X_{i}\right) \neq P\left(X_{j}\right) & \text {, If } Q_{i} \neq Q_{j} \\
0 & , \text { If } Q_{i}=Q_{j} \\
1, & P\left(X_{i}\right)=P\left(X_{j}\right), \text { If } Q_{i} \neq Q_{j}
\end{array}\right\} \quad i, j=1,2, \cdots, 14
$$

where $Q$ is the decision attribute and $P$ is the condition attribute.

Using the data listed in Table 4, a discernibility matrix is established via Eq.(10). The result is shown in Table 5.

In the discernibility matrix, "OR" operation is applied to each column and the results are manipulated with "AND" operation. The simplest reduced attributes or core attributes can be obtained with further logic operations. If we obtain only one individual attribute, then we can call this attribute "core attribute", otherwise we call these attributes as "simplest reduced attributes."

Accordingly, the discernibility relation of condition attributes can be reduced as

$$
(P 10) \vee(P 4 \vee P 6) \vee(P 1 \wedge P 2 \wedge P 3 \wedge P 7 \wedge P 8)
$$

Eventually, there are a total of ten possible combinations for the above condition attributes: (1)P1, P4, P10; (2) P2 , P4 , P10; (3) P3, P4, P10; (4) P4, P7, P10; (5) P4, T8, P10; (6) P1, P6, P10; (7) P2, P6, P10; (8) P3, P6, P10; (9) P6, P7, P10; (10) P6, P8, P10.

In other words, the resultant discernibility relation can be expressed as

$$
P i^{a} P j^{b} P k^{c} \rightarrow Q^{d}
$$

in which $P i, P j, P k$ may be any one of the above ten combinations $a, b, c$, and $d$ are "true"; and $Q$ means "YES."

There is one question remaining: If we use the above factor combination individually to perform its own linear regression model, then which combination can provide the best prediction result? The following section provides discussion of this issue. 
Table 5 Discernibility matrix (1 10 denote $\mathrm{P} 1 \sim \mathrm{P} 10$ respectively)

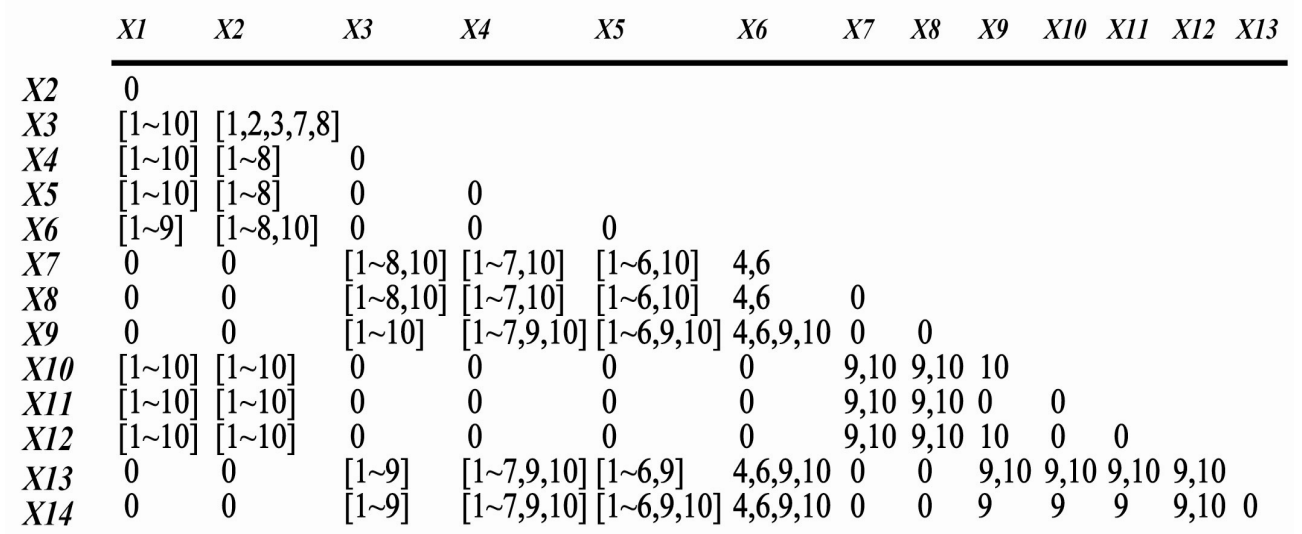

\subsection{Thermal Error Modeling}

\subsubsection{LR modeling}

Using all the above ten possible values of condition attributes combinations as dependent variables and the measured thermal deformation of the spindle nose in the $Z$-axis direction as the dependent variable, the linear regression modeling can be performed. The calculated result of least square error is shown in Table 6. It is seen that, among ten possible combinations, only the attributes combination $P 3, P 6$, and $P 10$ gives the smallest least square error (52.6) and the largest correlation coefficient (0.995). Hence, a LR thermal error model using this factor combination will lead to a better prediction compared to others. This LR equation is obtained as

$$
\delta_{Z}=-255.57+11.91(T 3)-3.57(T 6)+1.11(T 10)
$$

Table 6 Least square error and correlation coefficient of regression results for different temperature ascent combinations

\begin{tabular}{|c|c|c|}
\hline $\begin{array}{l}\text { Regression } \\
\text { Variables }\end{array}$ & $\begin{array}{l}\text { Correlation } \\
\text { Coefficient }\end{array}$ & $\begin{array}{l}\text { Least Square } \\
\text { Error }\end{array}$ \\
\hline$T 1 、 T 4 、 T 10$ & 0.979 & 237.9 \\
\hline$T 2 \cdot T 4 、 T 10$ & 0.990 & 111.7 \\
\hline$T 3 、 T 4 、 T 10$ & 0.995 & 57.54 \\
\hline$T 4 、 T 7 、 T 10$ & 0.989 & 121.95 \\
\hline$T 4 \cdot T 8 \cdot T 10$ & 0.992 & 88.97 \\
\hline$T 1 \cdot T 6 \cdot T 10$ & 0.968 & 356.97 \\
\hline$T 2 、 T 6 、 T 10$ & 0.991 & 95.86 \\
\hline$T 3 \cdot T 6 \cdot T 10$ & 0.995 & 52.6 \\
\hline$T 6 \cdot T 7 \cdot T 10$ & 0.975 & 281.08 \\
\hline$T 6 、 T 8 、 T 10$ & 0.981 & 218.59 \\
\hline
\end{tabular}

\subsubsection{ANFIS Modeling}

To understand the effectiveness of our proposed KRL model, a comprehensive artificial intelligence mapping scheme: the adaptive fuzzy inference system (ANFIS) ${ }^{\mathbf{( 2 0 , 2 7}}$ scheme is introduced for comparison.

From previous discussions, it is known that $T 3, T 6$, and $T 10$ have high linear rankings 
effects on the thermal drift of spindle nose in the $Z$-axis direction. Therefore, these three temperature ascents are adopted as fuzzy input variables of ANFIS.

Through table investigation method, choosing $T 3, T 6$, and $T 10$ as input variables and the displacement of spindle nose in the $Z$-axis direction as output variable, the fuzzy rule library can be constructed. The constructed rule library is a $7 \times 7 \times 7$ three dimensional array and in total thirteen rules can be obtained. For all measured data, $70 \%$ are used for training and $30 \%$ are used for checking. In training, a convergent criterion is set as error being less than or equivalent to $0.2 \mu \mathrm{m}$.

Through a compound learning algorithm ${ }^{\mathbf{2 8}}$, the membership functions describing the input and output variables are modified. After 23 epochs, the convergent condition is attained with a maximum error of $0.148 \mu \mathrm{m}$. The obtained control parameters of all membership functions before and after training are listed in Table 7. Using the modified ANFIS model, the maximum checking error is obtained as $0.168 \mu \mathrm{m}$.

Table 7 Membership functions (MF) before and after training

\begin{tabular}{|c|c|c|c|c|c|c|c|}
\hline \multirow[t]{2}{*}{ MF } & & \multicolumn{2}{|c|}{$T 3$} & \multicolumn{2}{|c|}{ T6 } & \multicolumn{2}{|c|}{$T 10$} \\
\hline & & $\begin{array}{l}\text { Initial } \\
\text { value }\end{array}$ & $\begin{array}{l}\text { Modified } \\
\text { value }\end{array}$ & $\begin{array}{l}\text { Initial } \\
\text { value }\end{array}$ & $\begin{array}{l}\text { Modified } \\
\text { value }\end{array}$ & $\begin{array}{l}\text { Initial } \\
\text { value }\end{array}$ & $\begin{array}{l}\text { Modified } \\
\text { value }\end{array}$ \\
\hline$S 3$ & $\begin{array}{l}\text { Mid. } \\
\text { Width }\end{array}$ & $\begin{array}{l}27.25 \\
0.875\end{array}$ & $\begin{array}{c}27.25 \\
0.875\end{array}$ & $\begin{array}{l}27.3 \\
1.187\end{array}$ & $\begin{array}{l}27.3 \\
1.187\end{array}$ & $\begin{array}{l}25.65 \\
0.2083\end{array}$ & $\begin{array}{l}25.66 \\
0.2848\end{array}$ \\
\hline$S 2$ & $\begin{array}{l}\text { Mid. } \\
\text { Width }\end{array}$ & $\begin{array}{l}29 \\
0.875\end{array}$ & $\begin{array}{l}29 \\
0.875\end{array}$ & $\begin{array}{c}29.68 \\
1.187\end{array}$ & $\begin{array}{c}29.67 \\
1.187\end{array}$ & $\begin{array}{l}26.07 \\
0.2083\end{array}$ & $\begin{array}{c}26.11 \\
0.2263\end{array}$ \\
\hline$S I$ & $\begin{array}{l}\text { Mid. } \\
\text { Width }\end{array}$ & $\begin{array}{c}30.75 \\
0.875\end{array}$ & $\begin{array}{c}30.75 \\
0.875\end{array}$ & $\begin{array}{c}32.05 \\
1.187\end{array}$ & $\begin{array}{c}32.05 \\
1.187\end{array}$ & $\begin{array}{l}26.48 \\
0.2083\end{array}$ & $\begin{array}{l}26.5 \\
0.1635\end{array}$ \\
\hline$M D$ & $\begin{array}{l}\text { Mid. } \\
\text { Width }\end{array}$ & $\begin{array}{l}32.5 \\
0.875 \\
\end{array}$ & $\begin{array}{l}32.5 \\
0.8745\end{array}$ & $\begin{array}{c}34.42 \\
1.187 \\
\end{array}$ & $\begin{array}{r}34.42 \\
1.187 \\
\end{array}$ & $\begin{array}{l}26.9 \\
0.2083 \\
\end{array}$ & $\begin{array}{c}26.96 \\
0.1245 \\
\end{array}$ \\
\hline B1 & $\begin{array}{l}\text { Mid. } \\
\text { Width }\end{array}$ & $\begin{array}{c}34.25 \\
0.875\end{array}$ & $\begin{array}{r}34.25 \\
0.89\end{array}$ & $\begin{array}{l}36.8 \\
1.187\end{array}$ & $\begin{array}{l}36.8 \\
1.195\end{array}$ & $\begin{array}{l}27.32 \\
0.2083\end{array}$ & $\begin{array}{l}27.3 \\
0.1354\end{array}$ \\
\hline$B 2$ & $\begin{array}{l}\text { Mid. } \\
\text { Width }\end{array}$ & $\begin{array}{l}36 \\
0.875\end{array}$ & $\begin{array}{l}36.02 \\
0.8706\end{array}$ & $\begin{array}{c}39.17 \\
1.187\end{array}$ & $\begin{array}{l}39.21 \\
1.215\end{array}$ & $\begin{array}{l}27.73 \\
0.2083\end{array}$ & $\begin{array}{l}27.73 \\
0.1949\end{array}$ \\
\hline B3 & $\begin{array}{l}\text { Mid. } \\
\text { Width }\end{array}$ & $\begin{array}{c}37.75 \\
0.875\end{array}$ & $\begin{array}{l}37.78 \\
0.8511\end{array}$ & $\begin{array}{c}41.55 \\
1.187\end{array}$ & $\begin{array}{c}41.58 \\
1.177\end{array}$ & $\begin{array}{l}28.15 \\
0.2083\end{array}$ & $\begin{array}{l}28.15 \\
0.2084\end{array}$ \\
\hline
\end{tabular}

\section{Model Comparison and Verification (real-cutting test)}

A new run of real-cutting tests is performed to compare the prediction ability of our proposed KRL model with ANFIS model and experimental results. The real-cutting condition was set as following: (1) end milling with 6 flutes and an $80 \mathrm{~mm}$ (diameter) cutter, (2) spindle speed $1000 \mathrm{rpm}$, (3) feed rate $1000 \mathrm{~mm} / \mathrm{min}$, (4) cutting depth $3.5 \mathrm{~mm}$, (5) reciprocating ZigZag cutting, and (6)cutting material: medium carbon steel 45C.

The temperature information read by the thermal sensor AD590J was sent to the temperature management program on a personal computer. The compensation value was testified in the cutting experiment for 1.5 hours. The comparison results are shown in Fig. 5. It is seen that thermal deformation of spindle nose in the Z-axis can be effectively reduced from $80 \mu \mathrm{m}$ to less than $8 \mu \mathrm{m}$. And, the maximum prediction errors for both models are less than 5\%. This phenomenon indicates that the prediction ability of the KRL scheme is as good as the ANFIS. This feature is very important for engineering application concerns. 


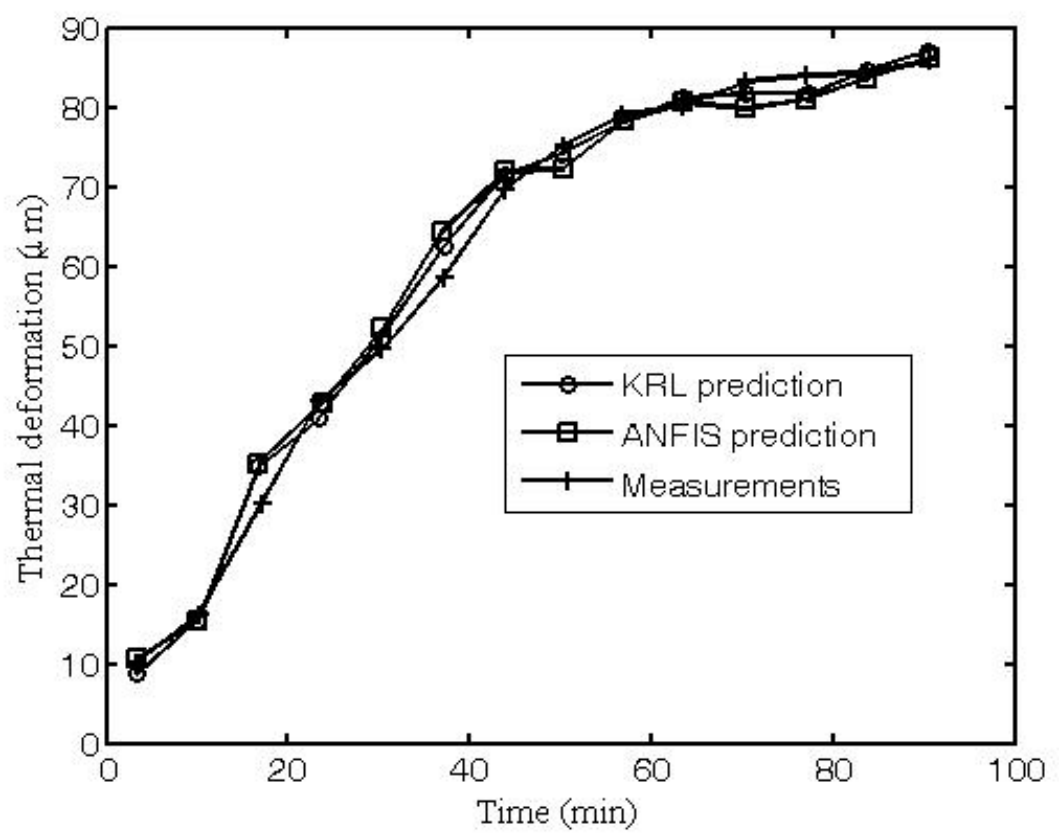

Fig. 5 Comparison of predictive and experimental results

\section{Conclusions}

A novel thermal error modeling technique including three knowledge discovery methods: KM, RS, and LR theories (called KRL model) were proposed in this study in order to map the temperature ascent against thermal drift of the machine tool in an accurate and economic way. The following conclusions are drawn by this study.

(1)The proposed KRL modeling scheme has the advantages of providing a transparent, simple and accurate thermal error model using as little measurement data as possible.

(2)Using the proposed KRL model, the thermal deformation of the spindle nose in the Z-axis direction can be compensated from $80 \mu \mathrm{m}$ to less than $8 \mu \mathrm{m}$.

(3)The KRL model has comprehensive mapping ability and its prediction accuracy is as good as that predicted by the ANFIS scheme. Predictions via the KRL and the ANFIS schemes deviates the experimental results (under a new test, real-cutting condition) less than $5 \%$.

(4)Such a KRL model is especially useful in a production environment in which engineers are facing the urgent requirement for a fast, transparent, easily-programmed and simply-installed modeling method.

(5)Our proposed model uses a C-type $\mathrm{CNC}$ machining center as application target. However, the proposed methodology of KRL model in this study can be applied to any type of CNC machining center with few adjustments needed in the modeling procedures.

\section{Acknowledgement}

The authors would like to thank the National Science Council of the Republic of China, Taiwan, for financially supporting this research under Contract No. NSC96-2622-E-275-003-CC3.

\section{References}

(1) M. Weck and L. Zangs, "Computing the thermal behavior of machine tools using the finite element method - Possibilities and limitations," Proceding of $16^{\text {th }}$ MTDR Conference, Vol. 16, pp185-194, 1975. 
(2) Z. Haitao, Y. Jianguo, and S. Jinhua, "Simulation of thermal behavior of a CNC machine tool spindle," International Journal of Machine Tools and Manufacture, Vol. 47, pp1003-1010, 2007.

(3) T. Moriwaki, "Thermal deformation and its on-line compensation of hydrostatically supported precision spindle," Annals of the CIRP, Vol. 37(12), pp283-286, 1988.

(4) S. Yang, J. Yuan, and J. Ni, “Accuracy enhancement of a horizontal machining center by real-time error compensation," Journal of Manufacturing Systems, Vol. 15(2), pp113-118, 1996.

(5) J. Janeczko, "Machine tool thermal distortion compensation," Proceedings of the $4^{\text {th }}$ Biennial International Machine Tool Technology Conference, 1988.

(6) S. Yang, et al., "Accuracy enhancement of a horizontal machining center by real-time error compensation,” Journal of Manufacturing System, Vol. 15 (2), pp113-118, 1996.

(7) J. S. Chen, "Computer-aided accuracy enhancement for multi-axis CNC machine tool," International Journal of Machine Tools and Manufactue, Vol. 35(4), pp593-605, 1995.

(8)J. S. Chen, "A study of thermally induced machine tool errors in real cutting conditions," International Journal of Machine Tools and Manufacture, Vol. 36(12), pp1401-1411, 1996.

(9)J. S. Chen and G. Chiou, "Quick testing and modeling of thermally-induced errors of CNC machine tools," International Journal of Machine Tools and Manufacture, Vol. 35(7), pp1063-1074, 1995.

(10)A. K. Srivastava, S. C. Veldhuis, and M. A. Elbestawi, "Modeling geometric and thermal errors in a five-axis CNC machine tool," International Journal of Machine Tools and Manufacture, Vol. 35(9), pp1321-1339, 1995.

(11)S. Li, Y. Zhang, and G. Zhang, "A study of pre-compensation for thermal errors of NC machine tools," International Journal of Machine Tools and Manufacture., Vol. 37 (12), pp1715-1719, 1997.

(12)J. S. Chen, "Fast calibration and modelling of thermally induced machine tool errors in real machining," International Journal of Machine Tools and Manufacture, Vol. 37 (2), pp159-169, 1997.

(13) Y. Wang, "Compensation for the thermal error of a multi-axis machining centre," ASME Trans. Joutnal of Materials Processing Technology, Vol. 75, pp45-53, 1998.

(14) M.Yang and J. Lee, "Measurement and prediction of thermal errors of a CNC machining centre using two spherical balls," ASME Trans. Journal of Materials Processing Technology, Vol. 75, pp180-189, 1998.

(15) C. D. Mize and J. C. Ziegert, "Neural network thermal error compensation of a machining center," Journal of the International Societies for Precision Engineering and Nanotechnology, Vol. 24, pp338-346, 2000.

(16) J. H. Lee, J. H. Lee, and S. H. Yang, "Thermal error modeling of a horizontal machining center using fuzzy logic stragedy," Journal of Manufacturing Processes, Vol. 3(2), pp120-127, 2001.

(17) J. H. Lee and S. H. Yangt, "Statistical optimization and assessment of a thermal error model for CNC machine tools," International Journal of Machine Tools and Manufacture, Vol. 42, pp147-155, 2002.

(18) Z. C. Du, et al., "Modeling approach of regression orthogonal experiment design for the thermal error compensation of a CNC turning center," Journal of Materials Processing Technology, Vol. 125, pp619-623, 2002.

(19) H. Yang and Jun Ni, "Dynamic Modeling for machine tool thermal error compensation," Journal of Manufacturing Science and Engineering, Vol. 125, pp245-254, 2003.

(20) K. C. Wang, P. C. Tseng, and K. M. Lin, "Thermal Error Modeling of a Machining Center Using Grey System Theory and Adaptive Network-Based Fuzzy Inference System," JSME International Journal, Series C, Vol. 49(4), pp1179-1187, 2006. 
(21) Y. Kang, C. W. Chang, Y. Huang, C. L. Hsu, and I. F. Nieh, "Modification of a neural network utilizing hybrid filters for the compensation of thermal deformation in machine tools," International Journal of Machine Tools and Manufacture, Vol. 47, pp376-387, 2007.

(22) R. Ramesh, M. A. Mannan, A. N. Poo, and S. S. Keerthi, "Thermal error measurement and modeling in machine tools. Part I. Influence of varing operating conditions," International Journal of Machine Tools and Manufacture, Vol. 43, pp391-404, 2003.

(23) R. Ramesh, M. A. Mannan, A. N. Poo, and S. S. Keerthi, "Thermal error measurement and modeling in machine tools. Part II. Hybrid Bayesian Network-support vector machine model," International Journal of Machine Tools and Manufacture, Vol. 43, pp405-419, 2003.

(24) J. B. Mac Queen, "Some methods for classification and analysis of multivariate observations," Proceedings of 5-th Berkeley Symposium on Mathematical Statistics and Probability, Berkeley, University of California Press, 1:281-297 V. Guralnik and G. Karypis, Workshop on Data Mining in Bioinformatics, 2001.

(25) Z. Pawlak, "Rough sets," International Journal of Computer and Information Sciences, Vol. 11, pp341-356, 1982.

(26) A. Skowron and A. Rauszer, "The discernibility matrics and functions in information systems," In: Slowinski R. (ed.): Intelligent decision support. Handbook of application and advances of the rough set theory, Kluwer, Dordrecht, pp331-362, 1992.

(27) J. S. Jang, "ANFIS: Adaptive-network-based fuzzy inference system," IEEE Transaction on Systems and Man Cybernetics, Vol. 23, pp665-685, 1993.

(28) K. C. Wang, P. C. Tseng, and K. M. Lin, "Thermal error modeling of a machining center using grey system theory and adaptive network-based fuzzy inference system," JSME International Journal, Series C, Vol. 49(4), 2006.

(29) W. Ziarko and N. Shan, "Discovering attribute relationships, dependencies and rules by using rough sets," Proceedings of the 28th Annual Hawaii International Conference on System Sciences (HICSS'95): pp293-299, 1995. 